\title{
Dual Reality: Merging the Real and Virtual
}

\author{
Joshua Lifton and Joseph A. Paradiso \\ MIT Media Lab
}

\begin{abstract}
This paper proposes the convergence of sensor networks and virtual worlds not only as a possible solution to their respective limitations, but also as the beginning of a new creative medium. In such a "dual reality," both real and virtual worlds are complete unto themselves, but also enhanced by the ability to mutually reflect, influence, and merge by means of sensor/actuator networks deeply embedded in everyday environments. This paper describes a full implementation of a dual reality system using a popular online virtual world and a humancentric sensor network designed around a common electrical power strip. Example applications (e.g., browsing sensor networks in online virtual worlds), interaction techniques, and design strategies for the dual reality domain are demonstrated and discussed.
\end{abstract}

Key words: dual reality, virtual worlds, sensor network

\section{Introduction}

At the heart of this paper is the concept of "dual reality," which is defined as an environment resulting from the interplay between the real world and the virtual world, as mediated by networks of sensors and actuators. While both worlds are complete unto themselves, they are also enriched by their ability to mutually reflect, influence, and merge into one another. The dual reality concept, in turn, incorporates two key ideas - that data streams from real-world sensor networks are the raw materials that will fuel creative representations via interactive media that will be commonly experienced, and that online 3D virtual worlds are an ideal venue for the manifestation and interactive browsing of the content generated from such sensor data streams.

In essence, sensor networks will turn the physical world into a palette, virtual worlds will provide the canvas on which the palette is used, and the mappings between the two are what will make their combination, dual reality, an art rather than an exact science. Of course, dual reality media will complement rather than replace other forms of media. Indeed, the end product, that which can be consumed and shared, is unlikely to outwardly resemble current forms of media, even if it is just as varied. Browsing the real world in a metaphorical virtual universe driven by a ubiquitous sensor network and unconstrained by physical boundaries approaches the concept of a digital "omniscience," where users can fluidly explore phenomena at different locations and scales, perhaps also interacting with reality through distributed displays and actuators. Indeed, a complete consideration of dual reality must also include the possibility of "sensor" data from the 


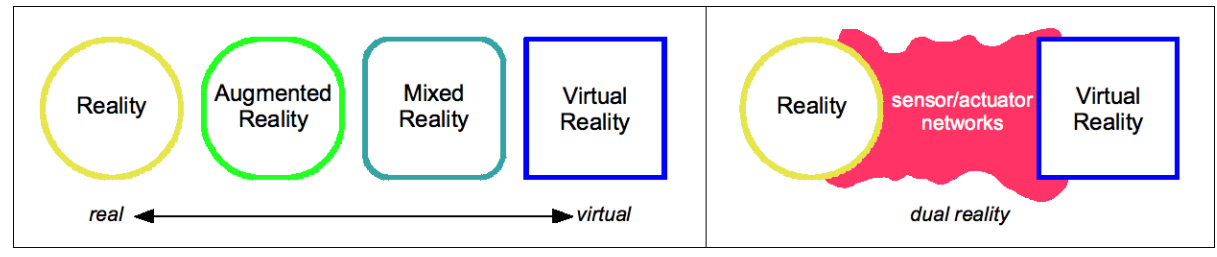

Fig. 1. An environmental taxonomy as viewed on the real-virtual axis (left). Sensor networks seamlessly merge real and virtual to form dual reality (right).

virtual world embodied in the real world. Insofar as technically feasible, dual reality is bi-directional - just as sensed data from the real world can be used to enrich the virtual world, so too can sensed data from the virtual world be used to enrich the real world.

Of the many axes along which various virtual worlds can be compared, the most relevant for this work is the real-virtual axis, which indicates how much of the constructed world is real and how much virtual. See Figure 1. A rough taxonomy can further compartmentalize the real-virtual axis into reality, which is simply life in the absence of virtual representations of the world; augmented reality, which has all aspects of reality, as well as an "information prosthetic" which overlays normally invisible information onto real objects 1, 2, mixed reality, which would be incomplete without both its real and virtual components, such as the partially built houses made complete with blue screen effects for use in military training exercises [3; and virtual reality, which contains only elements generated by a computer in an attempt to mimic aspects of the real world, as exemplified in some popular computer games [4]. Contrast this with the taxonomy given by Milgram and Kishino in [5].

Each of these environments represents what is supposed to be a single, complete, and consistent world, regardless of which components are real or virtual. Although this taxonomy can be successfully applied to most enhanced reality efforts, it does not address well the concept of dual reality, which comprises a complete reality and a complete virtual reality, both of which are enhanced by their ability to mutually reflect, influence, and merge into each other by means of deeply embedded sensor/actuator networks. See Figure 1.

\section{Background}

By their nature, sensor networks augment our ability to understand the physical world in ways beyond our innate capabilities. With sensor networks and a record of the data they generate, our senses are expanded in space, time, and modality. As with previous expansions of our ability to perceive the world, some of the first and perhaps in the long run most important upshots will be the stimulation of new creative media as artists working in dual reality strive to express sensed phenomena into strong virtual experiences. The work described here begins to explore directions for such self-expression as it takes shape in the interplay between sensor networks and virtual worlds. 
There is no definition of online virtual worlds that is both agreed upon and useful. The term itself is vague enough to encompass a full spectrum of technologies, from text-based multiple user domains (MUDs) originating in the late 1970s 6 to visually immersive online 3D games commercially available today [7. 8. This work primarily focuses on the concept of virtual world as introduced in science fiction works by authors such as William Gibson [9] and Neil Stephenson [10. This type of online virtual world is characterized by an immersive 3D environment, fluid interactions among inhabitants, and some level of ability for inhabitants to shape their environment. The goal may not be, and probably should not be, to replicate all aspects of the real world, but rather only those that facilitate the interaction in a virtual environment. In light of this, imbuing virtual worlds with the ability to sense aspects of the real world is a technique with significant potential.

The real world portions of this work use the 35-node Plug sensor network described in 11, 12, 13 and reviewed in a later section. The virtual world portions of this work focus exclusively on Second Life, an online virtual world launched in 2003 and today still maintained by Linden Lab [14. A comprehensive review of all online virtual worlds is beyond the scope of this work and better left to the several websites that specialize in such comparisons 7, 8, 15. Second Life was chosen because of its technical and other advantages in implementing many of the dual reality ideas explored here. For a more detailed introduction to Second Life, see Linden Lab's official guide book and the Second Life website [16, 14].

\subsection{Self-expression in Virtual Worlds}

Virtual worlds today are largely social in nature - people enter these worlds in order to meet other people and build connections with them through shared experiences. As in the real world, social interactions in virtual worlds revolve around self-expression. Taking Second Life as a representative example of the state-of-the-art in this respect, a resident of Second Life can express herself via the appearance and name of her avatar, the information revealed in her avatar's profile (favorite places, preferences, etc.), her avatar's scripted or explicitly triggered actions (dancing, laughing, running, etc.), text chat on public channels (received only by those nearby in the virtual world), text chat on private channels (received by a user-determined list of people regardless of their location in the virtual world), and live voice chat using a headset. A typical encounter when meeting another person for the first time, especially someone new to Second Life, revolves around explanations of how names and appearances were chosen, elaborations of details in avatar profiles, and exhibitions of clothing or animations.

A less explicit although arguably more compelling form of self-expression in Second Life is the ability to build objects, from necklaces to cars to castles, and imbue them with a wide range of behaviors. The skill level needed to do so, however, is on par with that needed to build compelling web sites. As such, this form of self-expression is limited to a small proportion of the total virtual world demographic. However, those who can build and script in Second Life can express themselves to a far wider audience than those who cannot. 
Compared to the real world, self-expression in Second Life and other virtual worlds is limited; missing are rich sources of information taken for granted in the real world, such as scent, body language, and the telltale signs of daily wear and tear. It's not that these sources of information were forgotten, just that they are difficult to emulate in meaningful ways in the virtual world. For example, virtual wind causes virtual trees to sway, a virtual sun and moon rise and set periodically, and virtual clouds form and disperse in Second Life, but there is no meaning or cause behind any of these phenomena and their effect on the virtual world is superficial at best. Overall, the demand for richer forms of selfexpression in virtual worlds is apparent. Data collected from real-world sensor networks can help meet this demand by importing into the virtual world the inherent expressiveness of the real world.

\subsection{The Vacancy Problem}

The vacancy problem is the noticeable and profound absence of a person from one world, either real or virtual, while they are participating in the other. Simply put, the vacancy problem arises because people do not currently have the means to be in more than one place (reality) at a time. In the real world, the vacancy problem takes the form of people appearing completely absorbed in themselves, ignoring everything else. In the virtual world, the vacancy problem

takes the form of virtual metropolises appearing nearly empty because there are not enough avatars to fill them. In part, this virtual vacancy is due to technical barriers preventing large numbers (hundreds) of people from interacting within the same virtual space. However, the vacancy problem will remain, even as processor speeds, network bandwidth, and graphics fidelity increase to overcome these technical difficulties. In a world nearly unconstrained by geography or physics, the currency of choice is people rather than real estate or possessions.

As of this writing, there are over 10 million registered Second Life accounts, but only about 50,000 users logged into Second Life at any given time [17, providing a population density of 10 people per square kilometer (vs. over 18,000 for real-world Manhattan).

The vacancy problem is a fundamental characteristic of today's virtual worlds. More closely linking the real world with the virtual world, as the dual reality concept suggests, can work to mitigate the vacancy problem - just as real cities require special infrastructure to allow for a high population density, so too will virtual cities. We can envision people continuously straddling the boundary between real and virtual through "scalable virtuality", where they are never truly offline, as sensor networks and mobile devices serve to maintain a continuous background inter-world connection (an early exploration of this idea was given in [18]). This can be tenuous, with virtual avatars passively representing some idea of the user's location and activity and the virtual world manifesting into reality through ambient display, or immersive, with the user fully engaged in manipulating their virtual presence. 


\subsection{Mapping Between Realities}

There are numerous challenges in designing exactly how the real and virtual will interact and map onto each other. A direct mapping of the real to virtual and virtual to real may not be the most appropriate. For example, the sensor data streams collected from a real person may be better mapped to the virtual land the person's avatar owns rather than to the avatar itself.

One possible mapping strategy is to shape the virtual world according to our subjective perceptions of the real world. In essence, the virtual world would be a reflection of reality distorted to match our mind's eye impressions as discerned by a network of sensors. For example, the buildings on a virtual campus could change in size according to the number of inhabitants and virtual corridors could widen or lengthen according to their actual throughput.

\subsection{Related Work}

Work that couples the real world with virtual worlds falls into several broad categories. There are several efforts to bring a virtual world into the real world by using positioning and proximity systems to cast real people as the actors of an otherwise virtual world, such as Human Pacman [19, Pac Manhattan [20], ARQuake [21], and DynaDOOM [22]. Such work remains almost exclusively within the realm of converting video games into live action games and, aside from location awareness, does not incorporate other sensing modalities. Magerkurth et al. provide a good overview of this genre of pervasive games, as well as other more sensor-rich but physically confined games 23. In an attempt to make Second Life more pervasive in the real world, Comverse has created a limited Second Life interface for cell phones 24. Virtual worlds are being used to involve citizens in the collaborative planning of real urban areas [25, although this type of system relies more on GIS data than sensor networks embedded in the environment. More advanced and correspondingly more expensive systems are used for military training [26]. Most of the systems mentioned above support only a handful of simultaneous users.

Among efforts to bring the real world into the virtual world, it is standard practice to stream audio and video from live real events, such as conferences and concerts, into Second Life spaces built specifically for those events [27. More ambitious and not as readily supported by existing technologies is the IBM UK Laboratories initiative in which the state of light switches, motorized blinds, the building's electricity meter, and the like in a real lab space are directly reflected and can be controlled in a Second Life replication [28. Similar efforts on a smaller scale include a general-purpose control panel that can be manipulated from both the real world and Second Life [29, and a homebrewed virtual reality wearable computer made specifically to interface to Second Life [30].

The convergence of Second Life, or something like it, with popular real-world mapping software to form a "Second Earth" has been broadly predicted [31. Uses of such a "hyper reality" include analyzing real-world data ("reality mining"), as was done in the Economic Weather Map project [32. Such ideas have appeared 
before as interactive art pieces. For example, the Mixed Realities juried art competition organized by Turbulence (a net art commissioning organization [33]) in collaboration with Ars Virtua (a media center and gallery within Second Life [34]) recognizes projects that mix various aspects of the real and virtual [35. Sensor network-enabled dual realities may naturally merge with or evolve from the life logging work pioneered by Gordon Bell [36, 37] and popularized by web applications such as MySpace [38, Facebook [39], and Twitter [40.

Central to the dual reality concept is the expressive and social intent of the participants, which separates dual reality from the broader field of information visualization [41, 42. For example, consider services like Google Maps [43] and Traffic.com [4], that visualizes traffic congestion in a large metropolitan area. Traffic information might be gathered from numerous sources, such as cell towers, arial imagery, or user input, and displayed in a variety of ways, such as on the web, in a 3D virtual environment, or text messaging. The primary use of this service is to allow participants to intelligently plan their daily commute. Although hardly social by most standards, this service does form a social feedback loop; a user of the service will change her route according to the data presented and in doing so change the nature of the data presented to the next user. However, the motivation or intent of the service is entirely devoid of self-expression, and therefore does not readily fall under the rubric of dual reality. Closer to dual reality is VRcontext's ProcessLife technology [45], which uses high-fidelity 3D virtual replicas of real environments to visualize and remotely influence industrial processes in real-time, though the potential for social interaction and rich metaphor appears low, as does the granularity of the sensor data visualizations.

\section{Design and Implementation}

\subsection{Real World Implementation}

This work utilizes the previously developed "Plug" sensor network comprising 35 nodes modeled on a common electrical power outlet strip and designed specifically for ubiquitous computing environments [11, 12, 13]. A Plug offers four standard US electrical outlets, each augmented with a precision transformer for sensing the electrical current and a digitally controlled switch for quickly turning the power on or off. The voltage coming into the Plug is also sensed. In addition to its electrical power sensing and control features, each Plug is equipped with two LEDs, a push button, small speaker, analog volume knob, piezo vibration sensor, microphone, light sensor, $2.4 \mathrm{GHz}$ low-power wireless transceiver, and USB 2.0 port. An external expansion port features a passive infrared (PIR) sensor motion sensor, SD removable memory card, and temperature sensor. All the Plug's peripherals are monitored and controlled by an Atmel AT91SAM7S64 microcontroller, which is based on the 32 -bit ARM7 core, runs at $48 \mathrm{MHz}$, and comes with $16 \mathrm{~KB}$ of SRAM and $64 \mathrm{~KB}$ of internal flash memory. Figure 2 shows Plug node with and without the external expansion. An extensive library of modular firmware can be pieced together into applications at compile time. 

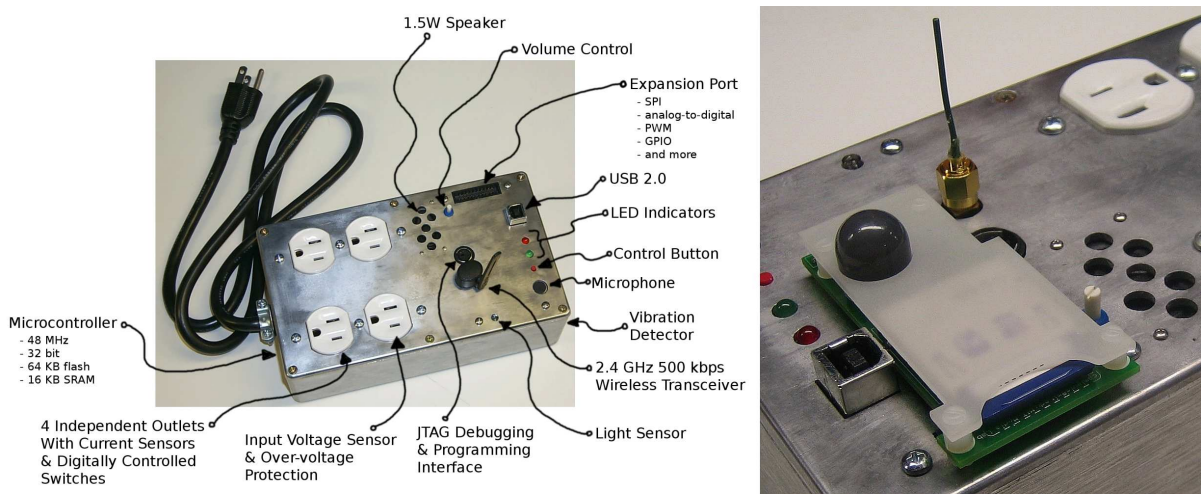

Fig. 2. A Plug sensor node with (right) and without (left) an external expansion.

\subsection{Virtual World Implementation}

The following sections describe objects or effects in the Second Life virtual world that were designed as an example of interfacing with the real world through sensor networks. Everything in Second Life exists as some combination of land, avatars, objects, and scripts. Land in Second Life is mapped directly to Linden Lab server resources, such as computing cycles, memory, and bandwidth. Avatars are the virtual manifestation of real people using Second Life. Objects are built from one or more primitive three-dimensional solids ("prims"), such as spheres, cubes, tori, and cones. A script is a program written in the Linden Scripting Language (LSL) and placed in an object to affect the object's behavior.

Data Ponds A single "data pond" is meant to be an easily distinguishable, locally confined representation of the sensor data from a single Plug node. See Figure 3. The data pond design consists of a cluster of waving stalks growing out of a puddle of water and an ethereal foxfire rising from among the stalks, as might be found in a fantastic swamp. The mapping between a Plug's sensor data and its corresponding data pond is easily understood once explained, but still interesting even without the benefit of the explanation. The particular mapping used is detailed in Table 1. The data ponds allowed sensed phenomena in the physical world to be efficiently browsed virtually, and proved effective, for example, in seeing at a glance which areas of our lab were more active than others. A real version of the data pond complements the virtual version. The real version follows the virtual's tentacle aesthetic by using a standard desk fan shrouded in a lightweight, polka dotted sheet of plastic. The air flow through the shroud and therefore the height, sound, and other idiosyncrasies of the shroud can be finely controlled by plugging the fan into the outlet of a Plug device and pulse width modulating the supply voltage accordingly. See Figure 3 .

Virtual Sensing Whereas real sensor networks capture the low-level nuance of the real world, virtual sensor networks capture the high-level context of the virtual world. For example, in reality, there are literally an infinite number of 

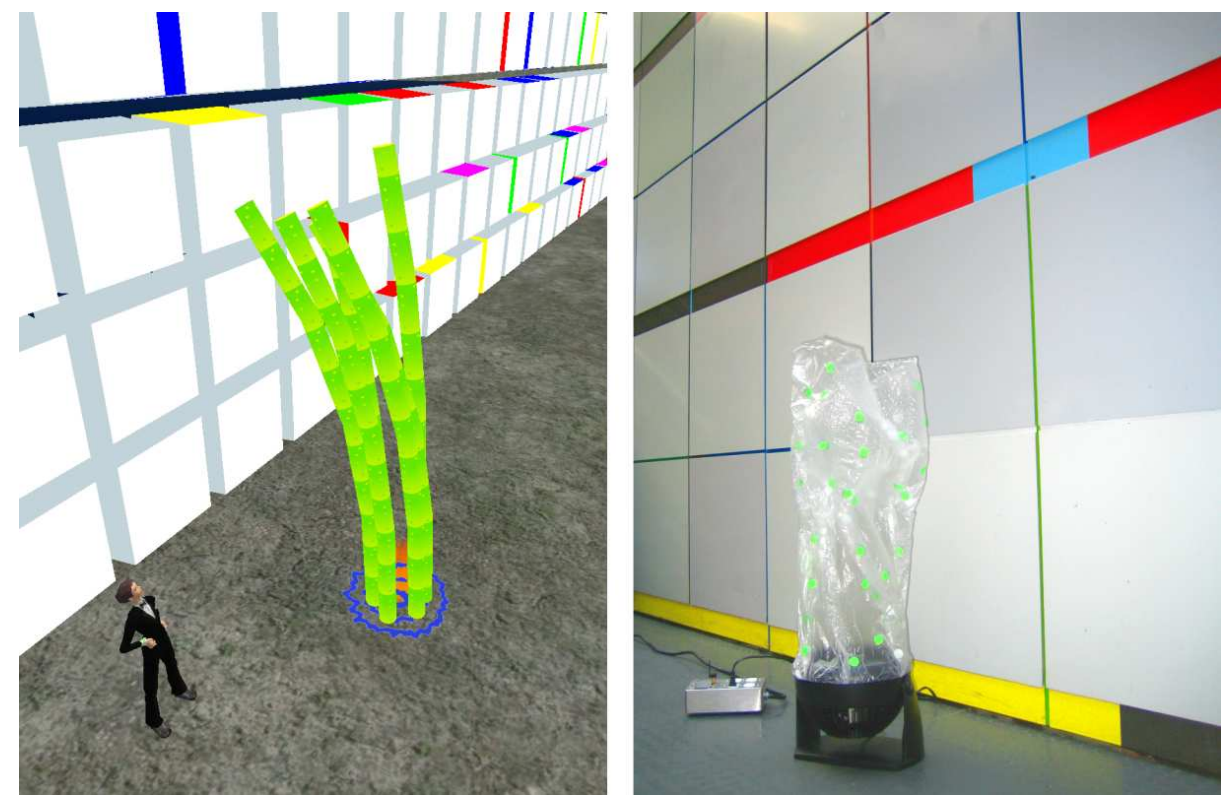

Fig. 3. A virtual data pond reflects real data near a virtual wall (left) and a real data pond reflects virtual data near a real wall (right).

\begin{tabular}{|l|l|l|}
\hline $\begin{array}{l}\text { Plug Sensor } \\
\text { Modality }\end{array}$ & $\begin{array}{l}\text { Data Pond } \\
\text { Attribute }\end{array}$ & Mapping \\
\hline \hline light & stalk length & $\begin{array}{l}\text { the stalk height is proportional to the maximum } \\
\text { light level over the most recent one-second window }\end{array}$ \\
\hline temperature & stalk color & $\begin{array}{l}\text { the color of the stalks varies linearly from blue to } \\
\text { yellow to red from } 18^{\circ} \mathrm{C} \text { to } 29^{\circ} \mathrm{C}\end{array}$ \\
\hline motion & stalk motion & $\begin{array}{l}\text { the stalks sway gently when no motion is detected } \\
\text { and excitedly when motion is detected over the } \\
\text { most recent one-second window }\end{array}$ \\
\hline electrical current & fire intensity & $\begin{array}{l}\text { the diameter of the water puddle is proportional } \\
\text { to the maximum sound level over the most recent } \\
\text { one-second window } \\
\text { to the total average absolute value of the electrical } \\
\text { current over the most recent one-second window }\end{array}$ \\
\hline
\end{tabular}

Table 1. The mapping from a real-world Plug's sensor data to its corresponding virtual data pond.

ways a person can touch a table, but in Second Life, there is exactly one. This work uses embedded and wearable virtual sensing schemes. The embedded sensing scheme entails seeding every object of interest in the virtual environment to be sensed with a script that detects when an avatar touches or otherwise interacts with the object and then reports back to a server external to Second Life with a full description of the interaction, including avatar position, speed, 


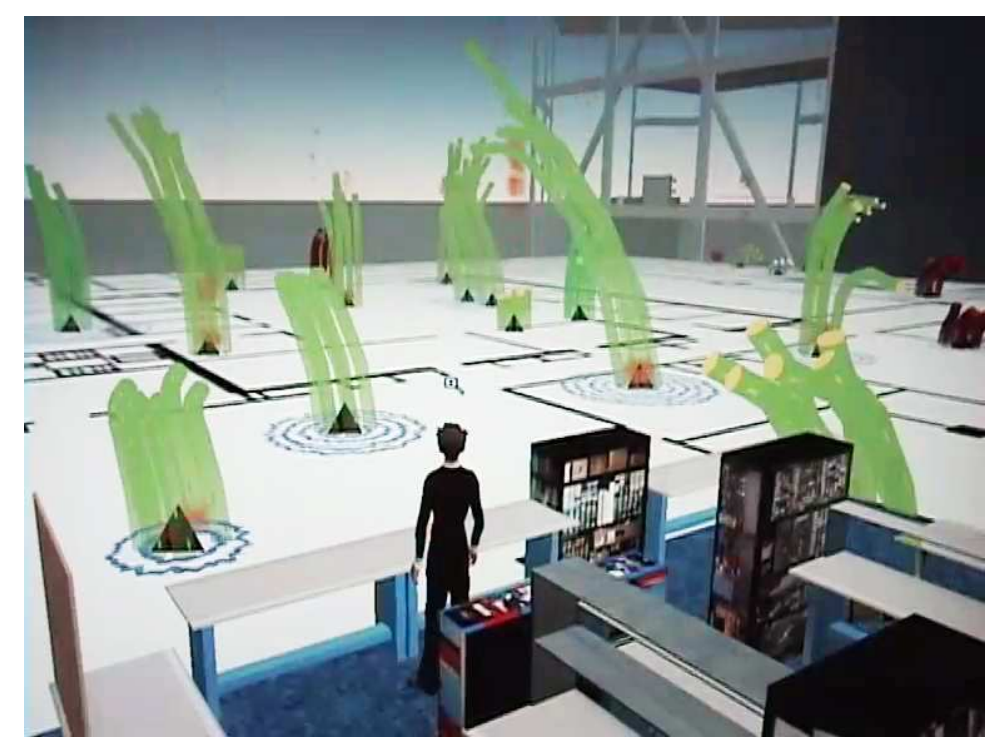

Fig. 4. Side view of the final implementation of Shadow Lab, which includes data ponds. A human-sized avatar is standing in the foreground - our particular labspace is rendered in detail, while the rest of the building was represented by a map. In the background are buildings belonging to unrelated neighbors.

rotation, and identity. The wearable sensing scheme requires each avatar in the region of interest to wear a sensing bracelet. The sensing bracelet reports back to the same external server every five seconds with a full description of its avatar's location, motion, and public channel chat. As incentive for avatars to wear the sensing bracelet, the bracelet also serves as an access token without which the avatar will be ejected from the region being sensed.

Shadow Lab Shadow Lab is a space in Second Life modeled after our real lab in which the Plug sensor network is deployed and exemplifies our real space to virtual space mapping. The primary feature of Shadow Lab is the to-scale two-dimensional floor plan of the third floor of our building. Only a small portion of the entire space is modeled in three dimensions. In part, this is due to the difficulty and resource drain of modeling everything in three dimensions. However, it is also a design decision reflecting the difficulty in maneuvering an avatar in a to-scale three dimensional space, which invariably feels too confining due to wide camera angles, quick movements, and the coarseness of the avatar movement controls in Second Life. Moreover, the two-dimensional design lends itself more readily to viewing the entire space at once and drawing attention to what few three-dimensional objects inhabit it. Figure 4 shows the latest version of Shadow Lab, which consists of the map of the lab, approximately 30 data ponds positioned on the map according to the positions of their corresponding Plugs in the real lab, and a video screen displaying a live video stream, when available, from a next-generation Tricorder [13] device equipped with a camera. 


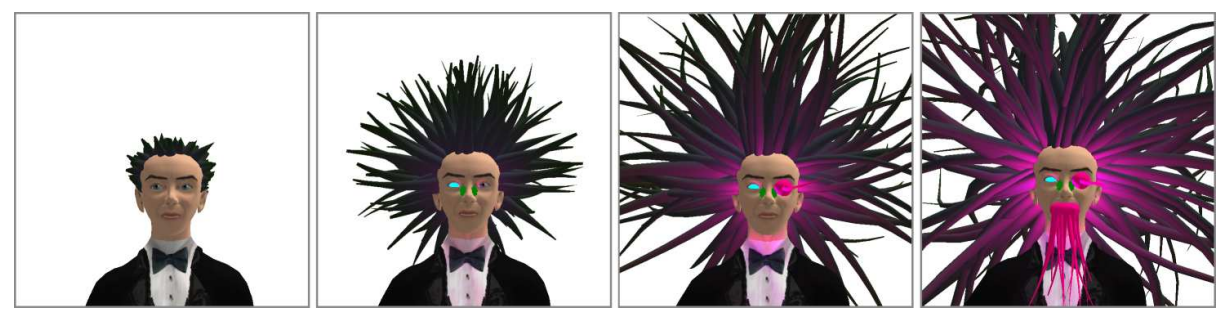

Fig. 5. Avatar metamorphosis (left to right) as real-world activity increases.

Metamorphosis The only unintentional body language exhibited in Second Life is the typing gesture avatars make when the user is typing a chat message, the slumped over sleeping stance assumed when the user's mouse and keyboard have been inactive for a preset amount of time, automatically turning to look at nearby avatars who have just spoken, and a series of stances randomly triggered when not otherwise moving, such as hands on hips and a bored slouch. All other body language and avatar actions must be intentionally chosen by the user. Clearly, there is room for improvement. Metamorphosis explores mapping real space to a virtual person. See Figure 5. In this prototype, the avatar begins as a typical human and transforms into a Lovecraftian alien according to several parameters drawn from the sensor streams of the Plug sensor network spread throughout the real building. While this particular example is outlandish and grotesque, in practice the mapping used in a metamorphosis is arbitrary, which is exactly its appeal as a method of self-expression - metamorphosis can be mapped to other arbitrary stimuli and unfold in any fashion.

Virtual Atrium The translation of our lab's atrium into Second Life attempts to retain that which is iconic about the original and at the same time take advantage of the freedom of the virtual world. See Figure6. The virtual atrium is defined by the intersection of two perpendicular walls of tile, one representing the total activity level of the real world as sensed by the Plug network and the one representing the total activity of the virtual world as sensed by the virtual sensing systems mentioned above. The physical extent and color scheme of the virtual atrium walls change accordingly. Each tile has a blank white front face, four colored sides, and a black back face. Touching a tile will cause it to flip over, at which point the black back face comes to the front and changes to reveal a hidden movie or image. All tiles in a given wall share the same image or movie when flipped, although the exact image or movie displayed is variable.

Dual Reality Open House At the time of this writing, the state-of-the-art in large events that bridge the real and virtual worlds amounts to what is essentially video conferencing between a real auditorium and a virtual auditorium [46]. As a prototype demonstration of moving beyond this by employing sensor networks, a dual reality open house was constructed to introduce residents of Second Life to the lab and visitors of the lab to Second Life. The dual reality open house premiered at a one-day technical symposium and held in the atrium of our 

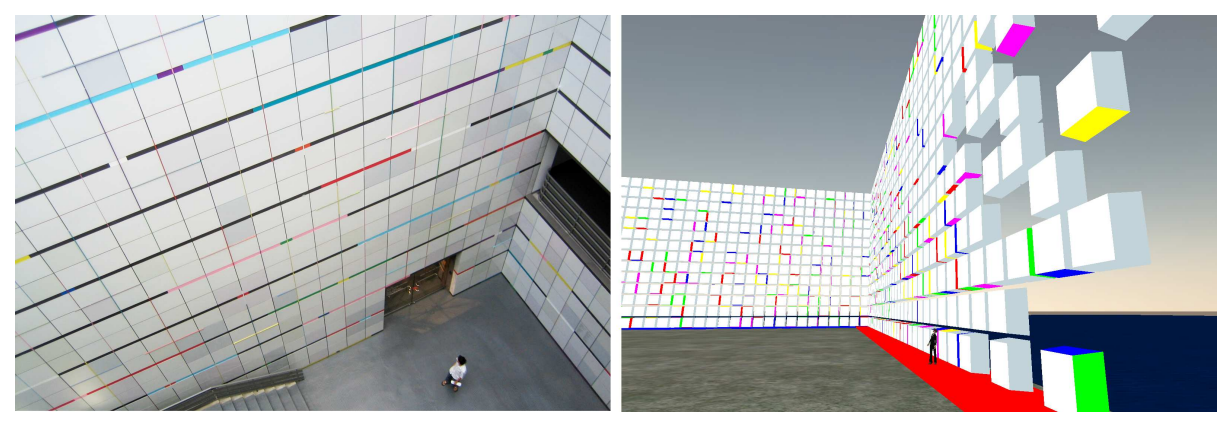

Fig. 6. The real lab atrium (left) and the virtual version (right). A real person and an avatar show their respective scales.

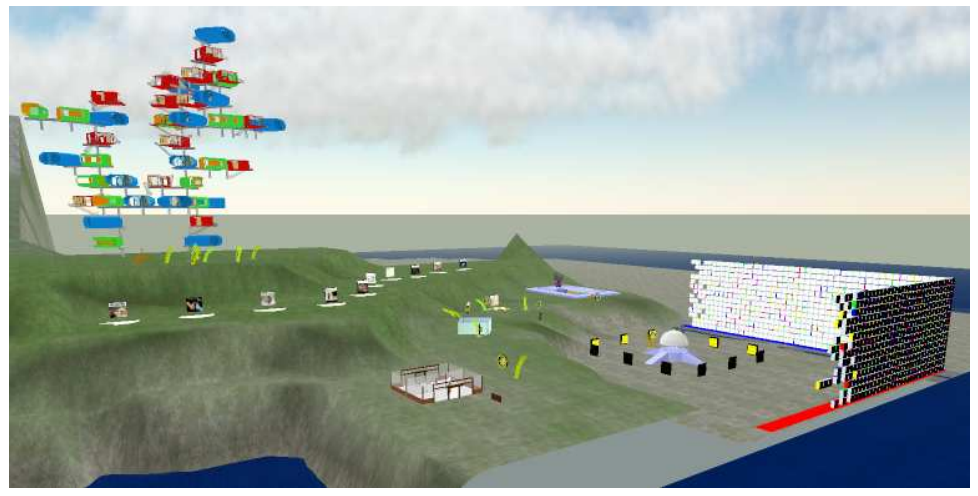

Fig. 7. Side view of the Ruthenium region.

lab [47. The real portion of the event consisted of talks and panel discussions in the building's main auditorium, interspersed with coffee breaks and standup meals in the atrium among tables manned by lab students demonstrating various lab projects related to virtual worlds. The virtual portion of the open house was located in a typical 256-meter by 256-meter region of Second Life 48 called "Ruthenium." The server running the Ruthenium region is limited to 40 simultaneous avatars and 15,000 simultaneous prims. In preparation for the open house, Ruthenium was terraformed and filled with static information kiosks and live demonstrations of various projects from around the lab. More details about the projects displayed can be found in [11. The virtual atrium described in 3.2 framed the space where the virtual portion of our event took place. Data ponds and an avatar metamorphosis were featured as well. See Figure 7. The entire Ruthenium region employs the virtual sensing schemes described earlier.

\section{Dual Reality Event and Discussion}

The dual reality open house described earlier has the potential to explore the real data and virtual data collection systems. (See [12, 11] for more detailed 

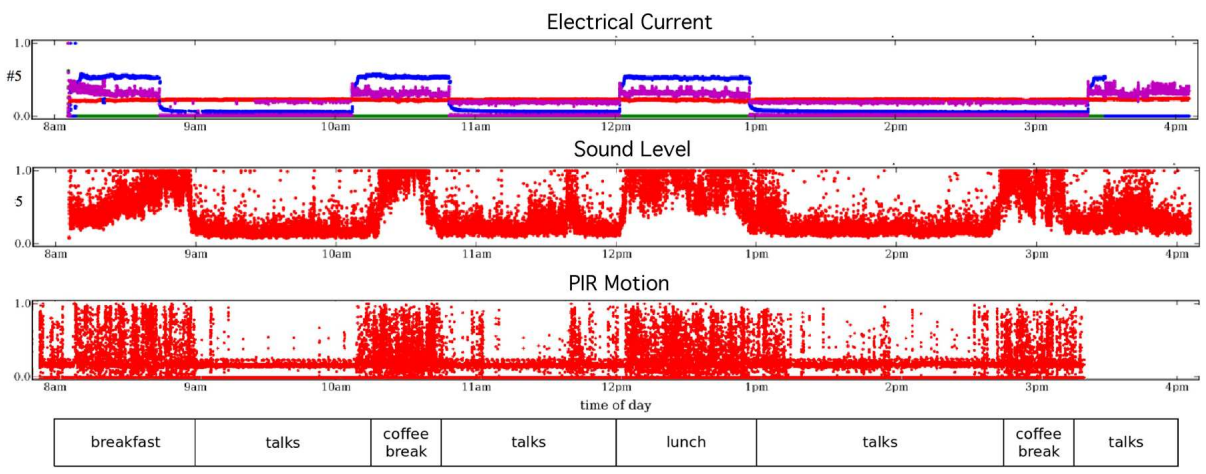

Fig. 8. Electrical current, sound level, and motion versus time from a typical Plug node during the dual reality open house.

evaluations of the Plug sensor network.) Sensor data from both the real world and virtual world were collected during the day-long event. The real-world data originated from the Plug sensor nodes used throughout the real lab atrium at the various open house demo stations. Motion, sound, and electrical current data from a typical Plug are shown in Figure 8. Also collected but not shown here are data for each Plug's light, voltage, vibration, and temperature sensors. The virtual-world data originated from the virtual sensing system previously detailed as deployed throughout the virtual portion of the dual reality open house described earlier. Such an extensive data set from a single event spread across both real and virtual worlds had not previously been collected.

By the nature of the event and its presentation in each world, very little correlation between the real and virtual data was expected. However, each data set does speak to how people interact within each world separately and what the possibilities are for using data from one world in the other. The real-world sound and motion data shown in Figure 8 clearly follows the structure of the event as attendees alternate between the atrium during break times and the auditorium during the conference talks - the auditorium is noisier during breaks, during which demo equipment was also generally switched on and people are moving around the demos. On the other hand, the light data (not shown) indicate physical location more than attendee activity - direct sunlight versus fluorescent lights versus LCD projector light. See [1] for more detail.

Of the various data collected from the virtual world during the day-long event, Figure 9 shows the distribution over time of touch events (avatars touching a virtual object equipped with the virtual embedded sensing system) and avatar movement events (the virtual wearable sensing system checks if its avatar is moving approximately once per second) collected from 22 avatars, of which 16 chose to wear the access bracelet virtual sensing system. Due to a network glitch, data collected from the virtual sensing system started being logged at approximately $11 \mathrm{AM}$ rather than at $8 \mathrm{AM}$, when the event actually started. The spike of avatar movement at around noon is likely due to the pause in the live video stream from the auditorium when the talks broke for lunch, thus giv- 

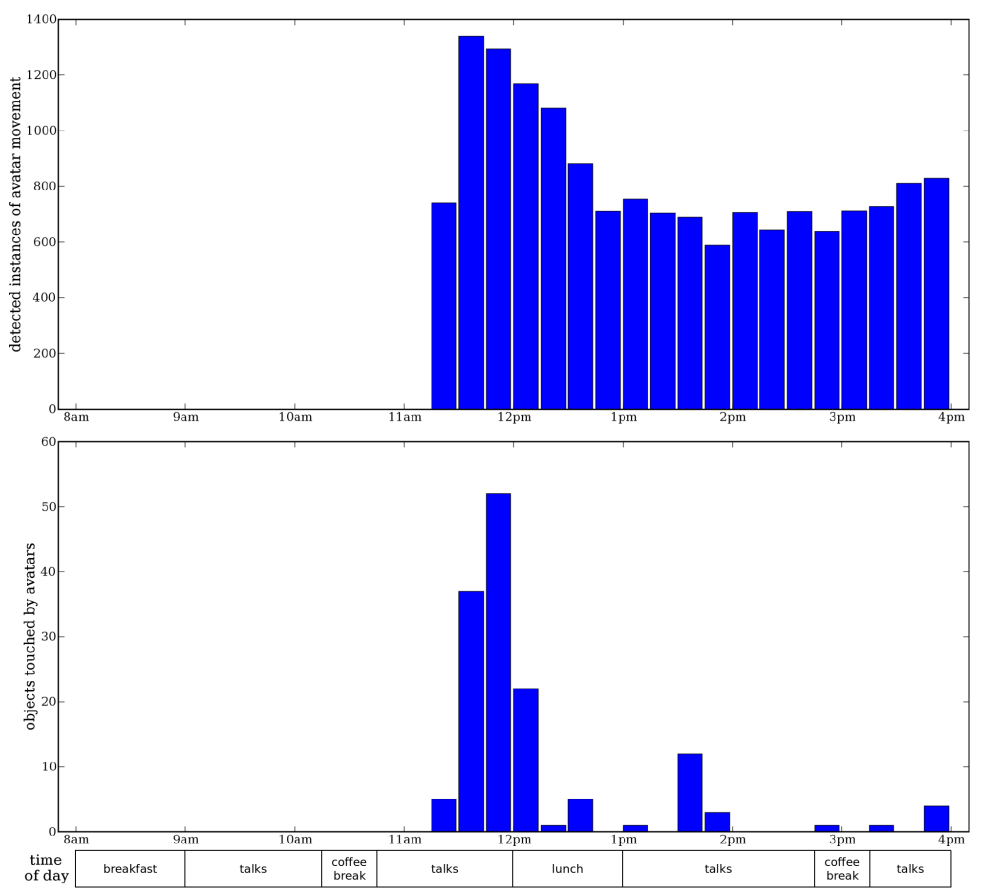

Fig. 9. Avatar movement and interaction during the dual reality open house.

ing avatars watching the video stream incentive to move to another location to interact with other aspects of the virtual space. The relatively constant motion thereafter might indicate the exploratory nature of the participants and/or the space. Of all avatar-object interactions, $83 \%$ were between an avatar and a virtual atrium wall tile, that displayed the live video feed from the real auditorium.

This trial could have been improved in several respects. For example, the number of virtual attendees could have been increased with better advertising. Also (and most crucially), a stronger connection between real and virtual premises could have been made and "connectedness" metrics formulated and tested. These are being addressed in another dual reality event that we are hosting soon.

\subsection{Discussion}

In a completely fabricated virtual world, the entropy of a real-world data stream can dramatically alter the virtual ambiance. Certainly, a cleverly utilized pseudorandom number generator could do the same, but meaning derives more from perception than from the underlying mechanism, and it is much easier to weave a story from real data than from pseudo-random numbers. The act of weaving a story from sensor data is essentially the act of designing and implementing a mapping from data to a real or virtual manifestation of the data. A successful story must be meaningful to tell as well as to hear, and using sensor data 
grounded in either the real or virtual world helps achieve this. In essence, the act of creation must be as gratifying as the act of consumption.

The creative aspects of dual reality, the mapping of real or virtual sensor data to some manifestation, will likely follow the trend of another recent medium blogs. While blogs have allowed some creative geniuses an outlet and given them a wide, appreciative, and well-deserved audience, the quality of most blogs, at least as a consumptive medium, is far below previous mass media standards. Of course, their quality as a creative medium and the value they bring to their creators in that regard far exceed previous standards by virtue of their relatively low barrier to entry alone. These trends will be exaggerated in the context of dual reality for two reasons. First, the medium is much richer, involving virtual $3 \mathrm{D}$ worlds and complex social interactions and is therefore accessible to a wider audience. Second, once the mapping of data to manifestation is set, the act of creation is nearly automatic (sitting somewhere between an interactive installation and a performance) and therefore a wider range of talent will participate. In short, the worst will be worse and the best will be better, a hallmark of successful mass media. As with other creative media, virtuosity will still play a critical role in dual reality, namely in the conception, implementation, and honing of the specific mappings between sensor data and their manifestations. These ideas are further discussed in 49 .

While mapping sensor data to manifestation may be at the highest level of the dual reality creative process, once the mappings are in place, people can still intentionally express themselves in many ways, depending on the exact nature of the mapping. The evolution of emoticons in text messages is one example of such expression using a current technology. Another is the habit of maintaining an active online presence, such as used in Internet messaging clients, by jogging the computer's mouse occasionally. In the same way, users of dual reality environments will modify their behavior so as to express themselves through the medium.

\section{Conclusion}

Various technologies have fundamentally altered our capacity to consume, share, and create media. Most notably, television and radio made consumption widespread and the Internet made sharing widespread. In comparison, creation of media is still difficult and limited to a small subset of the population. The promise of dual reality is to use sensor/actuator networks as a generative tool in the process of transforming our everyday experiences in the real world into content shared and experienced in the virtual world. Just as the data created by a movie camera are shared and consumed in a theater, the data collected from sensor networks will be shared and consumed in virtual worlds. This holds the potential to revolutionize sensor network browsing, as participants fluidly explore metaphoric representations of sensor data - similarly, virtual denizens can manifest into real spaces through display and actuator networks. If sensor networks are the palette, then virtual worlds are the canvas that usher in a new form of mass media. 


\section{References}

1. S. Feiner et al. Knowledge-based Augmented Reality. Comm. of the ACM, 36(7):53-62, 1993.

2. Sportvision. Virtual Yellow 1st and Ten. http://www.sportvision.com/, 1998.

3. F. S. Dean Jr. et al. Mixed Reality: A Tool for Integrating Live, Virtual \& Constructive Domains to Support Training Transformation. In Proc. of the Interservice/Industry Training, Simulation, and Education Conference (I/ITSEC), 2004.

4. Electronic Arts. SimCity. http://simcity.ea.com/, 2007.

5. P. Milgram and F. Kishino. A taxonomy of mixed reality visual displays. IEICE Trans. of Information Systems, E77-D(12), December 1994.

6. H. Rheingold. The Virtual Community: Homesteading on the Electronic Frontier. Addison-Wesley, 1993.

7. R. Good. Online Virtual Worlds: A Mini-Guide. http://www.masternewmedia.org/virtual_reality/virtual-worlds/ virtual-immersive-3D-worlds-guide-20071004.htm April 2007.

8. B. Book. Virtual Worlds Review. http://www.virtualworldsreview.com/ February 2006.

9. W. Gibson. Neuromancer. Ace Books, 1984.

10. N. Stephenson. Snow Crash. Bantam Books, 1992.

11. J. Lifton. Dual Reality: An Emerging Medium. Ph.D. Dissertation, M.I.T., Dept. of Media Arts and Sciences, September 2007.

12. J. Lifton et al. A Platform for Ubiquitous Sensor Deployment in Occupational and Domestic Environments. In Proc. of the Sixth Int'l Symposium on Information Processing in Sensor Networks (IPSN), pages 119-127, April 2007.

13. J. Lifton et al. Tricorder: A mobile sensor network browser. In Proc. of the ACM CHI 2007 Conference - Mobile Spatial Interaction Workshop, April 2007.

14. Linden Lab. Second Life. http://www.secondlife.com, 2003.

15. J. Lifton. Technology Evaluation for Marketing \& Entertainment Virtual Worlds. Electric Sheep Co. Report http://www.electricsheepcompany.com/publications/, 2008.

16. M. Rymaszewski et al. Second Life: The Official Guide. Wiley, 2007.

17. Linden Lab. Economic Statistics. http://secondlife.com/whatis/economy_stats.php, 2007.

18. M. Musolesi et al. The Second Life of a Sensor: Integrating Real-world Experience in Virtual Worlds using Mobile Phones. In Fifth ACM Workshop on Embedded Networked Sensors (HotEmNets), June 2008.

19. A. D. Cheok et al. Human Pacman: A Mobile Entertainment System with Ubiquitous Computing and Tangible Interaction over a Wide Outdoor Area. In Fifth Int'l Symposium on Human Computer Interaction with Mobile Devices and Services (Mobile HCI), pages 209-223, September 2003.

20. PacManhattan. http://pacmanhattan.com, 2004.

21. B. Thomas et al. ARQuake: An Outdoor/Indoor Augmented Reality First Person Application. In Fourth Int'l Symposium on Wearable Computers (ISWC'00), 2000.

22. G. Sukthankar. The DynaDOOM Visualization Agent: A Handheld Interface for Live Action Gaming. In Workshop on Ubiquitous Agents on Embedded, Wearable, and Mobile Devices (Conference on Intelligent Agents 85 Multiagent Systems), July 2002 . 
23. C. Magerkurth et al. Pervasive Games: Bringing Computer Entertainment Back to the Real World. ACM Computers in Entertainment, 3(3), July 2005.

24. W. Roush. New Portal to Second Life: Your Phone. Technology Review, http://www.technologyreview.com/Infotech/18195/, 2007.

25. J. MacIntyre. Sim Civics. Boston Globe: http: //www.boston.com/news/globe/ideas/articles/2005/08/07/sim_civics/, August 2005.

26. W. Miller. Dismounted Infantry Takes the Virtual High Ground. Military Training Technology, 7(8), December 2002.

27. D. Jansen. Beyond Broadcast 2007 - The Conference Goes Virtual: Second Life. http://www . beyondbroadcast.net/blog/?p=37, 2006.

28. IBM. Hursley Island. http://slurl.com/secondlife/Hursley/0/0/0/, 2007.

29. ciemaar. Real Life Control Panel for Second Life. http://channel3b.wordpress. com/2007/01/24/real-life-control-panel-for-second-life/, 2007.

30. P. Torrone. my wearable computer - snowcrash. http://www.flickr.com/photos/pmtorrone/sets/1710794/, January 2006.

31. W. Roush. Second Earth. Technology Review, 110(4):38-48, July/August 2007.

32. G. Boone. Reality Mining: Browsing Reality with Sensor Neworks. Sensors Magazine, 21(9), September 2004.

33. Turbulence. http://www.turbulence.org/, 2007.

34. Ars Virtua. http://arsvirtua.org/, 2007.

35. Turbulence. Mixed Realities Commissions. http://transition.turbulence.org/comp_07/awards.html, 2007.

36. G. Bell. A Personal Digital Store. Comm. of the ACM, 44(1):86-91, January 2001.

37. J. Gemmell et al. MyLifeBits: A Personal Database for Everything. Comm. of the ACM, 49(1):88-95, January 2006.

38. MySpace. http://www.myspace.com/ 2007.

39. Facebook. http://www.facebook.com/, 2007.

40. Twitter. http://twitter.com/ 2007.

41. E. R. Tufte. The Visual Display of Quantitative Information. Graphics Press, 1983.

42. C. Chen. Information Visualisation and Virtual Environments. Springer-Verlag, 1999.

43. Google. Google Maps. http://maps.google.com 2007.

44. Navteq. Traffic.com. http://www.traffic.com, 2007.

45. VRcontext. ProcessLife. http://www.vrcontext.com/ February 2009.

46. S. Verbeck. Founder and CEO of The Electric Sheep Company. Personal comm. via e-mail, July 9, 2007.

47. IBM. Virtual Worlds: Where Business, Society, Technology, \& Policy Converge. http://www.research.ibm.com/research/press/virtualworlds_agenda.shtml, June 15, 2007.

48. J. Lifton. Media Lab Dual Reality Open House. http://slurl.com/secondlife/Ruthenium/0/0/0/, 2007.

49. J. Lifton, M. Laibowitz, D. Harry, N. Gong, M. Mittal, and J.A. Paradiso. Metaphor and Manifestation: Cross Reality with Ubiquitous Sensor/Actuator Networks. IEEE Pervasive Computing Magazine, summer 2009. 